\title{
Use of a Laser Engraver in Relocations and Sample Preparation for SEM and Light Microscope Analysis.
}

\author{
Abigail P. Lindstrom ${ }^{1}$, Nicholas W.M. Ritchie ${ }^{1}$ and Michael Mengason ${ }^{1}$ \\ 1. National Institute of Standards and Technology, Materials Measurement Laboratory, Gaithersburg, \\ MD, 20899, USA
}

In a multi-instrument laboratory, it is common to analyze micro-features on multiple different microscopes using a variety of electron and light optical technologies. Relocation is the ability to take a sample from one microscope to another and to identify the same micro-features quickly and efficiently. We use fiducial marks in our relocations - distinctive marks that can be readily identified using various different microscope technologies. The fiducial marks are located in each microscope and coordinate transformations defined that map a feature's stage coordinate in one microscope to equivalent coordinates in another microscope. For a flat sample, 3 fiducial marks are sufficient to define a transform consisting of five degrees of freedom - a 2-dimension translation, a 2-dimension scale change and a rotation. We investigated a new way of making fiducial marks using a laser engraver. The laser engraver, an Epilog Fusion M2 [1], which works like a printer in Microsoft Windows-compatible applications, can be used to make sophisticated patterns easily and reproducibly. These patterns can be used in various different ways to assist with sample preparation including placing fiducial marks.

Using a vector-mode graphics program, it is possible to draw sophisticated patterns that can be "printed" using a high energy pulsed laser on various different types of opaque substrates including pyrolytic carbon planchets and silicon wafers. Complex features are constructed by driving the laser head over the sample to print larger features (raster mode) or single pit-wide lines (vector mode.) The pit size depends upon the laser power and the substrate but is of the order of $35 \mu \mathrm{m}$ as shown in Figure 1a. We found that diagonal vectors were not perfectly straight but had a ripple on the order of a $50 \mu \mathrm{m}$ as shown in Figure $1 \mathrm{~b}$.

To test the feasibility of using the engraver for relocations, we used the engraver to mark a 1 ” silicon substrate with 3 distinctive fiducial marks at approximately $120^{\circ}$ intervals with an $\times$-shape where the two arms of the $\times$ meet at a single pit. To make the fiducials distinctive, we surrounded the $\times$ es with a circle, a square and a Greek cross approximately $1 \mathrm{~mm}$ in size. We added twelve additional $\times$ marks in random locations around the mount. Using the TESCAN MIRA-3, we marked the locations of a feature at the center of the fiducials and a feature at the center of the 12 random $\times$ es. The mount was then removed from the instrument and replaced at a random offset and rotation. We located the fiducial marks again and used NIST Relocate, an internally developed relocation program [2], to calculate the transform, and applied it to the original coordinates of the 12 randomly placed $\times$ es. We were able to relocate the $\times$ es to within 5 $\mu \mathrm{m} \pm 2 \mu \mathrm{m}$. We tried the same relocation with a light microscope, Olympus BH-2, at $5 \times$ and found we could relocate the test $\times$ es to $26 \mu \mathrm{m} \pm 13 \mu \mathrm{m}$. Some of the difference in accuracy was due to the differences in magnification between the 2 techniques, but the majority of the difference was due to difficulties with centering objects in a window, which is much easier with a SEM.

We expect that the laser engraver will become an important tool in our sample preparation process. The flexibility of the device will permit us to add fiducial marks, scale markers and unique labels to many of our common substrate materials. Furthermore, the laser can be used to add marks directly to some flat samples like cross-sections of rocks, engineered items or epoxy mounts. 
[1] Certain products, either public domain or commercial, are identified in this paper. Identification of such products does not imply recommendation or endorsement by NIST, nor does it imply that the identified product is the best available.

[2] NIST Relocate is available as component in the NIST DTSA-II installation. NIST DTSA-II is available for free download from www.cstl.nist.gov/div837/837.02/epq/dtsa2/index.html.

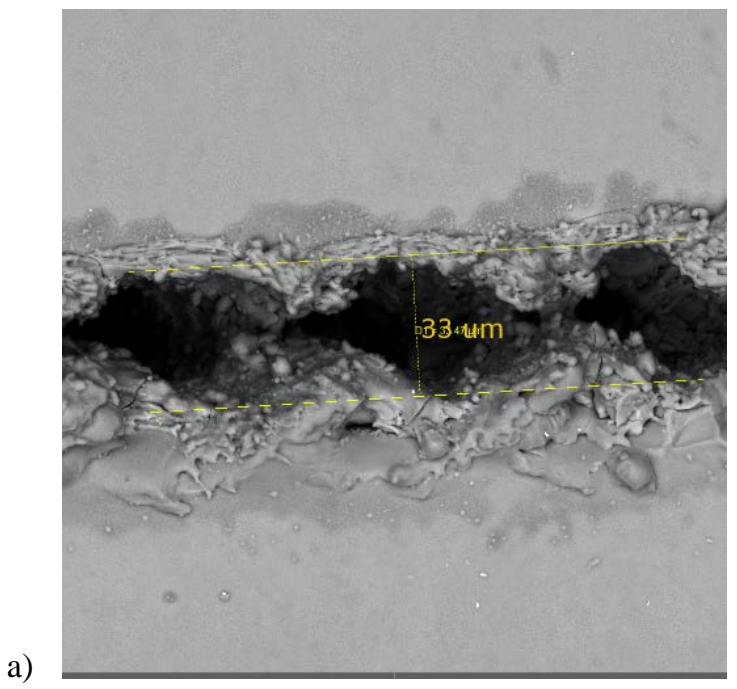

a)

Figure 1. Straight and diagonal lines as etched on a silicon substrate. The image in figure 1a shows the width of the pits and the image in figure $1 \mathrm{~b}$ the variation in the lengths of the diagonals.

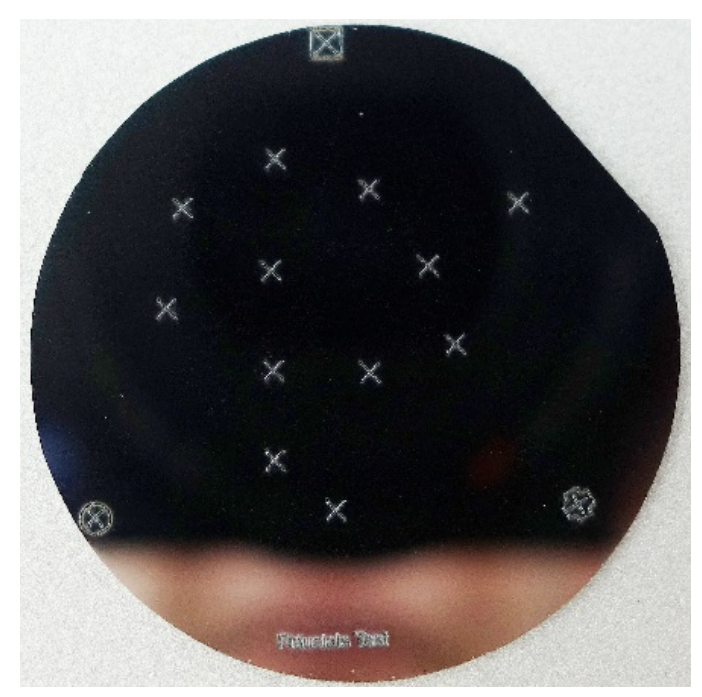

Figure 2. The Si substrate used in the relocation test showing the fiducial marks and the 12 relocation test points. 